\title{
Nano-engineered high-confinement AIGaAs waveguide devices for nonlinear photonics
}

Pu, Minhao; Zheng, Yi; Stassen, Erik; Kamel, Ayman Nasar; Bony, Pierre-Yves; Ottaviano, Luisa; Semenova, Elizaveta; Yvind, Kresten

\section{Published in:}

Proceedings of SPIE

Link to article, DOI:

$10.1117 / 12.2307114$

Publication date:

2018

Document Version

Publisher's PDF, also known as Version of record

Link back to DTU Orbit

Citation $(A P A)$ :

Pu, M., Zheng, Y., Stassen, E., Kamel, A. N., Bony, P-Y., Ottaviano, L., Semenova, E., \& Yvind, K. (2018). Nano-engineered high-confinement AlGaAs waveguide devices for nonlinear photonics. In Proceedings of SPIE (Vol. 10672, pp. 106721R-106721R-7). SPIE - International Society for Optical Engineering. Proceedings of SPIE - The International Society for Optical Engineering https://doi.org/10.1117/12.2307114

\section{General rights}

Copyright and moral rights for the publications made accessible in the public portal are retained by the authors and/or other copyright owners and it is a condition of accessing publications that users recognise and abide by the legal requirements associated with these rights.

- Users may download and print one copy of any publication from the public portal for the purpose of private study or research.

- You may not further distribute the material or use it for any profit-making activity or commercial gain

- You may freely distribute the URL identifying the publication in the public portal 


\section{Nano-engineered high-confinement AlGaAs waveguide devices for nonlinear photonics}

Minhao Pu, Yi Zheng, Erik Stassen, Ayman Nasar Kamel, Pierre-Yves Bony, et al.

Minhao Pu, Yi Zheng, Erik Stassen, Ayman Nasar Kamel, Pierre-Yves Bony, Luisa Ottaviano, Elizaveta Semenova, Kresten Yvind, "Nanoengineered high-confinement AIGaAs waveguide devices for nonlinear photonics," Proc. SPIE 10672, Nanophotonics VII, 106721R (4 May 2018); doi: $10.1117 / 12.2307114$

SPIE. Event: SPIE Photonics Europe, 2018, Strasbourg, France 


\title{
Nano-engineered high-confinement AlGaAs waveguide devices for nonlinear photonics
}

\author{
Minhao Pu*, Yi Zheng, Erik Stassen, Ayman Nasar Kamel, Pierre-Yves Bony, Luisa Ottaviano, \\ Elizaveta Semenova, and Kresten Yvind
}

\author{
DTU Fotonik, Department of Photonics Engineering, Technical University of Denmark, \\ Building 343, DK-2800 Lyngby, Denmark
}

\begin{abstract}
The combination of nonlinear and integrated photonics enables applications in telecommunication, metrology, spectroscopy, and quantum information science. Pioneer works in silicon-on-insulator (SOI) has shown huge potentials of integrated nonlinear photonics. However, silicon suffers two-photon absorption (TPA) in the telecom wavelengths around $1550 \mathrm{~nm}$, which hampers its practical applications. To get a superior nonlinear performance, an ideal integrated waveguide platform should combine a high material nonlinearity, low material absorption (linear and nonlinear), a strong light confinement, and a mature fabrication technology. Aluminum gallium arsenide (AlGaAs) was identified as a promising candidate for nonlinear applications since 1994. It offers a large transparency window, a high refractive index $(\mathrm{n} \approx 3.3)$, a nonlinear index $\left(n_{2}\right)$ on the order of $10^{-17} \mathrm{~m}^{2} \mathrm{~W}^{-1}$, and the ability to engineer the material bandgap to mitigate TPA. In spite of the high intrinsic nonlinearity, conventional deep-etched AlGaAs waveguides exhibit low effective nonlinearity due to the vertical low-index contrast. To take full advantage of the high intrinsic linear and nonlinear index of AlGaAs material, we reconstructed the conventional AlGaAs waveguide into a high index contrast layout that has been realized in the AlGaAs-on-insulator $(\mathrm{AlGaAsOI})$ platform. We have demonstrated low loss waveguides with an ultra-high nonlinear coefficient and high $Q$ microresonators in such a platform. Owing to the high confinement waveguide layout and state-of-the-art nanolithography techniques, the dispersion properties of the AlGaAsOI waveguide can be tailored efficiently and accurately by altering the waveguide shape or dimension, which enables various applications in signal processing and generation, which will be reviewed in this paper.
\end{abstract}

Keywords: Integrated optics; Nonlinear optics; Nonlinear materials; Parametric processes; Optical signal processing; Optical communication; Frequency comb; Supercontinuum generation

\section{INTRODUCTION}

Aluminum gallium arsenide $\left(\mathrm{Al}_{x} \mathrm{Ga}_{-x} \mathrm{As}\right)$ is a promising candidate for integrated nonlinear photonics due to its large second-order $\left(\chi^{(2)}\right)$ and third-order $\left(\chi^{(3)}\right)$ nonlinearities [1], [2]. The exhibits a wide transparency window and its material bandgap can be engineered by changing the aluminum composition $(x)$ to avoid two-photon absorption (TPA) at telecom wavelengths $(\sim 1.55 \mu \mathrm{m})$, which has been identified as the most detrimental effects for an efficient nonlinear process. The linear refractive index of $\mathrm{Al}_{x} \mathrm{Ga}_{1-x} \mathrm{As}$ is similar to silicon, which makes it possible to make high confinement waveguide. Over the past two decades, significant efforts have been made to realize efficient nonlinear processes in AlGaAs waveguides. As high quality AlGaAs film can only be epitaxial grown on lattice-matched GaAs substrate, whose refractive index is higher than $\mathrm{AlGaAs}$, the conventional $\mathrm{AlGaAs}$ waveguides are realized by deep etching a thick $\mathrm{AlGaAs}$ layer stack. The layer stack typically consists of $\mathrm{Al}_{x} \mathrm{Ga}_{1-x} \mathrm{As}$ layers with different aluminum compositions $(x)$ where the index of the waveguiding layer is slightly larger than that of its adjacent top and bottom layers [3]-[5]. The fabrication of such waveguides with very high and narrow mesa structures is challenging, and increased sidewall roughness as the etch depth is increased is typically seen which severely affects the linear loss and, thus, the nonlinear efficiency. Although extensive optimization on the fabrication process has been made over past years [6]-[9] and lowloss $(\sim 1 \mathrm{~dB} / \mathrm{cm})$ deep-etched AlGaAs waveguides are achieved by using a resist reflow technique [9]. However, because of the high aspect ratio of the waveguide, more advanced designs than straight waveguides such as microring resonators with integrated waveguides are still challenging to realize with high performances. The highest achieved quality factors (Q) of deep-etched AlGaAs microring resonators are at a very low level $\left(<10^{4}\right)$ [10]-[12]. In addition, the low vertical index-contrast of such $\mathrm{AlGaAs}$ waveguides severely limit its effective nonlinearity in spite of a high material nonlinearity. The weak light confinement also makes the device dispersion less sensitive to the waveguide dimension

*mipu@fotonik.dtu.dk; phone 4545256358

Nanophotonics VII, edited by David L. Andrews, Angus J. Bain, Jean-Michel Nunzi, Andreas Ostendorf, Proc. of SPIE Vol. 10672, 106721R · C 2018 SPIE · CCC code: 0277-786X/18/\$18 · doi: 10.1117/12.2307114 
change and thus difficult to engineer the device dispersion. To fully take advantage of the material nonlinearity, a highindex contrast waveguide layout is desired to enhance the light confinement and thus the light-matter interaction. We propose and realize the AlGaAs-on-insulator (AlGaAsOI) platform [13], [14] where a thin AlGaAs layer resides on an insulator material, allowing device patterning using an easy shallow etching process.

\section{ALUMINUM GALLIUM ARSENIDE-ON-INSULATOR PLATFORM}

We developed wafer bonding and substrate removal process to fabricate AlGaAsOI wafers for nonlinear waveguide devices. Compared with the conventional low-index contrast AlGaAs waveguides, the surface roughness control become more critical due to stronger light fields at the waveguide-cladding interfaces. Targeting at applications at telecom wavelength range, we applied polymer-bonding process for AlGaAs-on-silica wafers where a high refractive index semiconductor substrate is used and a buffered oxide layer with typical several micron thickness is used in between to avoid light leakage. However, such a platform is not suitable for applications at the mid-infrared wavelength range even though AlGaAs is transparent because the insulator layer material (glass) has a strong material absorption at wavelengths longer than $2.5 \mu \mathrm{m}$ [15]. To extend the operation wavelength range of AlGaAs material, sapphire can be used as the substrate material. We also developed $\mathrm{Al}_{2} \mathrm{O}_{3}$-assisted direct wafer bonding (DWB) process for fabricating AlGaAs-onsapphire wafers [16]. The fabrication processes for both AlGaAsOI wafers are shown in Figure 1. First, an epitaxial stack, including $\mathrm{AlGaAs}$ and a dual-etch-stop layer, was grown in a low-pressure Metalorganic vapor phase epitaxy (MOVPE) reactor Emcore D125 on (100) GaAs substrates. For AlGaAsOI wafers with silica as the insulator layers, a 90-nm thick benzocyclobutene (BCB) layer is used as the adhesive layer for bonding between the AlGaAs and carrier wafers. For $\mathrm{AlGaAsOI}$ wafers with sapphire as the substrate, we deposit very thin (a few nanometers) $\mathrm{Al}_{2} \mathrm{O}_{3}$ on both AlGaAs and sapphire wafers by using atomic layer deposition (ALD) before annealing. Note that, surface cleanliness and roughness are critical for DWB. A less than $0.5 \mathrm{~nm}$ roughness is required to achieve a high quality bonding. The annealing temperature was optimized for a strong bonding strength in both cases. And the GaAs substrate is removed in two wet etching steps, including a fast etch step with a sulfuric acid/hydrogen peroxide solution $\left(\mathrm{H}_{2} \mathrm{SiO}_{4}: \mathrm{H}_{2} \mathrm{O}_{2}\right)$ and a seven-hour slow etch step in a selective citric acid/hydrogen peroxide solution $\left(\mathrm{C}_{6} \mathrm{H}_{8} \mathrm{O}_{7}: \mathrm{H}_{2} \mathrm{O}_{2}\right)$ [14]. After removing the etch-stop InGaP layers in $\mathrm{HCl}$ solution, the $\mathrm{AlGaAsOI}$ wafers are ready for device fabrications. Compared with $\mathrm{AlGaAs}-$ on-silica wafers, the AlGaAs-on-sapphire ( $\mathrm{AlGaAsOS})$ wafers not only have a wider operation wavelength range but also offer a better thermal conductivity $\left(25-50 \mathrm{Wm}^{-1} \mathrm{~K}^{-1}\right)$, which is an order of magnitude larger than that of silica. However, sample cleaving for AlGaAsOS samples is more challenging than that for standard AlGaAsOI samples because of the sapphire substrate. In this paper, we only discuss experimental results for AlGaAs-on-silica devices at telecom wavelengths.

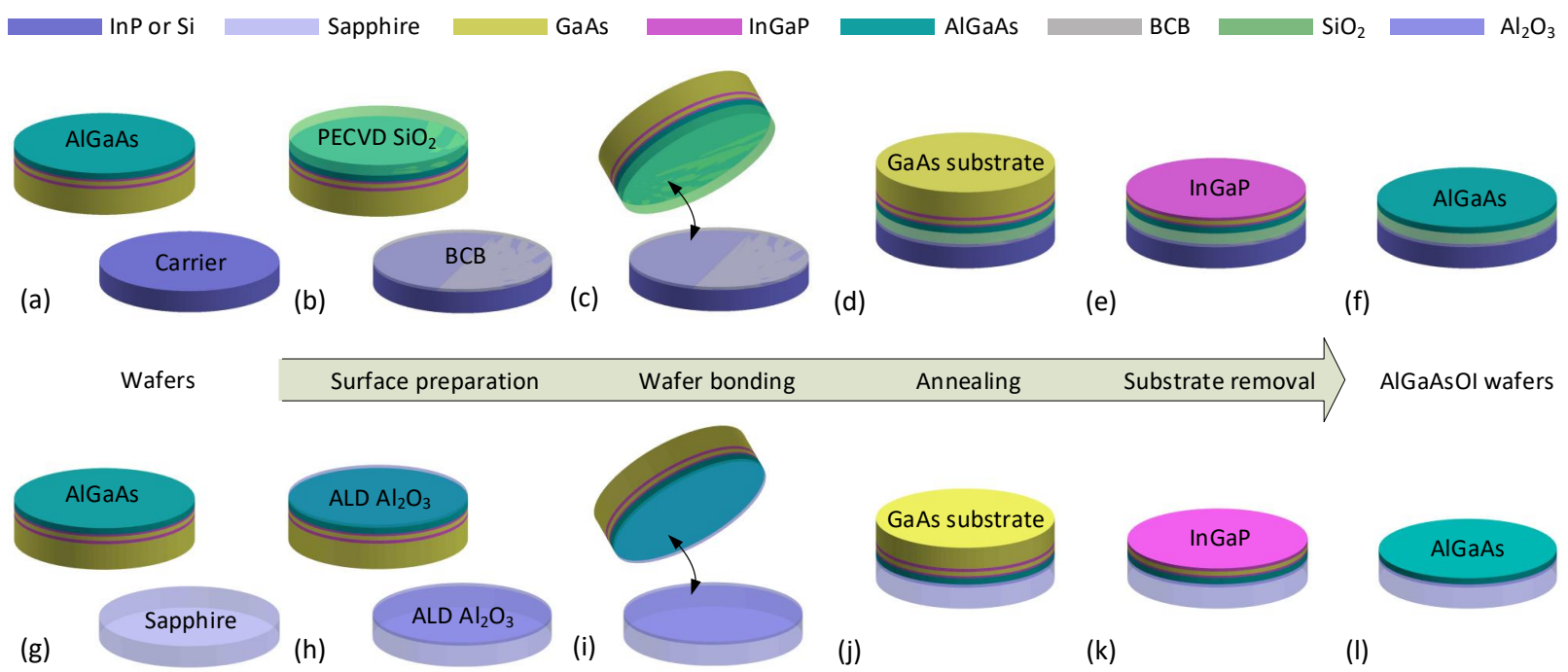

Figure 1 Fabrication process for AlGaAsOI wafers with different bottom insulator materials: silica (a-f) and sapphire (g-l). The process starts with a GaAs wafer with epitaxial grown AlGaAs top layer and a carrier wafer (semiconductor (a) or sapphire (g)). For AlGaAsOI wafer with silica as the insulator layer, a $\mathrm{SiO} 2$ (BCB) layer is deposited on the $\mathrm{AlGaAs}$ (carrier) wafer (b). For AlGaAsOI wafer with sapphire as the substrate, very thin Al2O3 layers are deposited on both AlGaAs and sapphire carrier wafers (h). The two wafers are then bonded (c,i) and annealed (d,j). The GaAs substrate is wetetched with the AlGaAs layer being protected by the etch-stop layer (e,k). After wet etching the etch-stop layer, AlGaAsOI wafers are ready for device fabrication (f, 1 ). 


\section{LINEAR LOSS PERFORMANCE FOR WAVEGUIDE DEVICES}

Figure 2(a) shows the schematic drawing of the AlGaAsOI waveguide. As shown by the simulated electrical field distribution, the light can be confined in a sub-micron cross-section waveguide core. As the nonlinear parameter $(\gamma)$ is highly dependent on the waveguide effective modal are $\left(A_{\text {eff }}\right)$ as expressed by $\gamma=2 \pi n_{2} /\left(\lambda A_{\text {eff }}\right)$ [17], an ultra-high effective nonlinearity up to $660 \mathrm{~W}^{-1} \mathrm{~m}^{-1}$, which is orders of magnitude higher than those of highly nonlinear fibers (HNLFs) and $\mathrm{Si} 3 \mathrm{~N} 4$ waveguides, can be obtained for an AlGaAsOI waveguide with a sub-micron cross-section dimension [13]. High index-contrast waveguide device performance is typically limited by linear losses induced by light scattering from surface roughness, especially the sidewall roughness. Device patterning processes including electron beam lithography (EBL) and dry etching have been optimized to get smooth patterning. Hydrogen silsesquioxane (HSQ, Dow Corning FOX-15) was used as the electron beam resist and since its index is similar to glass, we kept it on top of the AlGaAs layer. Figure 2(b) shows a fabricated waveguide with smooth sidewalls just after dry-etching process and Figure 2(c) shows the cross-section of an $\mathrm{AlGaAsOI}$ waveguide after the cladding glass deposition. We characterize the loss of AlGaAsOI waveguides with the same height $(300 \mathrm{~nm})$ but with different widths. As shown in Figure 2(d), the linear loss is highly dependent on the waveguide width; the linear loss reduces from $2.3 \mathrm{~dB} / \mathrm{cm}$ to $1 \mathrm{~dB} / \mathrm{cm}$ when the waveguide width is increased from $400 \mathrm{~nm}$ to $1000 \mathrm{~nm}$. The low linear loss will ensure a longer effective interaction length for efficient nonlinear processes and high Q microresonators are expected once the narrow gaps between microresonator and bus waveguide can be fabricated.
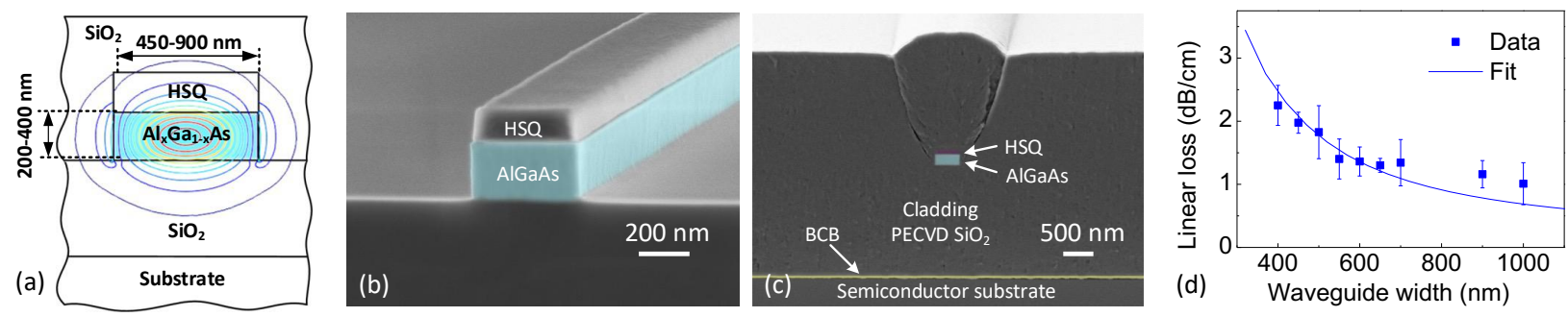

Figure 2 (a) Schematic drawing of the AlGaAsOI waveguide where simulated electrical field distribution for the fundamental TE mode is superimposed. (b) Scanning electron microscopy (SEM) picture of a fabricated waveguide just after dry-etching process. (c) SEM side-view picture of a fabricated waveguide after the cladding silica is deposited. The AlGaAs layer is denoted by the artificial blue color. (d) Measured linear losses for AlGaAsOI waveguides with a 300-nm height but with different waveguide widths.
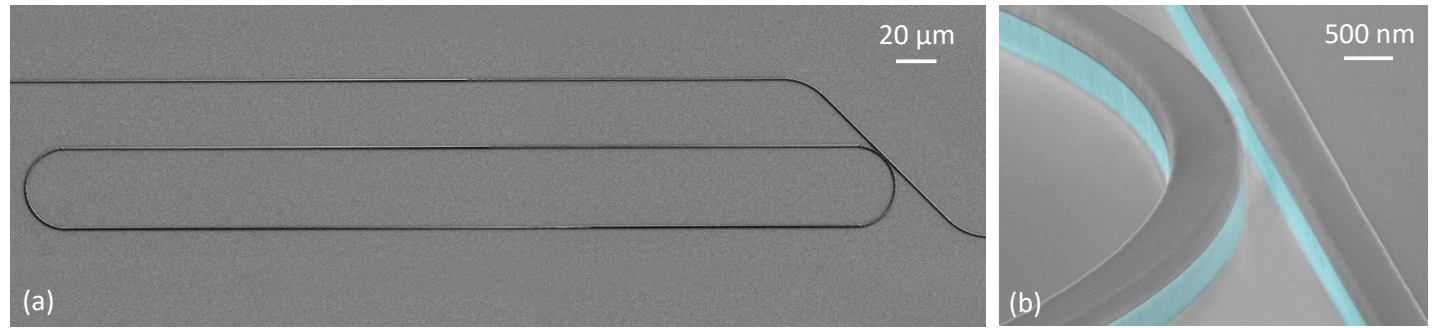

Figure 3 SEM pictures: (a) Top-view of an 810- $\mu$ m long AlGaAsOI microresonator. (b) Bird's-eye view of the coupling region of the $\mathrm{AlGaAsOI}$ microresonator. The $\mathrm{AlGaAs}$ material is indicated by the blue color.

High quality factor $(Q)$ microresonators are important components for enhancing the efficiency of nonlinear processes such as four-wave mixing (FWM) and optical parametric oscillation (OPO). We fabricated microresonators with a cavity length of $810 \mu \mathrm{m}$ as shown in Figure 3(a). Since only shallow etching is required to define the AlGaAsOI devices, microresonators with integrated bus waveguides can be easily defined as shown in Figure 3(b). To extract the Q of different microresonators, we measured the transmission spectrum of those microresonators and Figure 4 shows the measured spectra for fundamental TE mode of microresonators with different cross-section dimensions. A multi-pass 
EBL process was applied here in order to improve the patterning of curved structure [14]. The intrinsic Qs we can extracted from the measurement are $\sim 4.9 \times 105$ and $\sim 6.9 \times 105$, respectively, for $630-\mathrm{nm}$ and $900-\mathrm{nm}$ wide waveguides. The demonstrated $\mathrm{Q}$ of the $\mathrm{AlGaAsOI}$ microresonators is more than one order of magnitude higher than that has been previously reported [10]-[12].
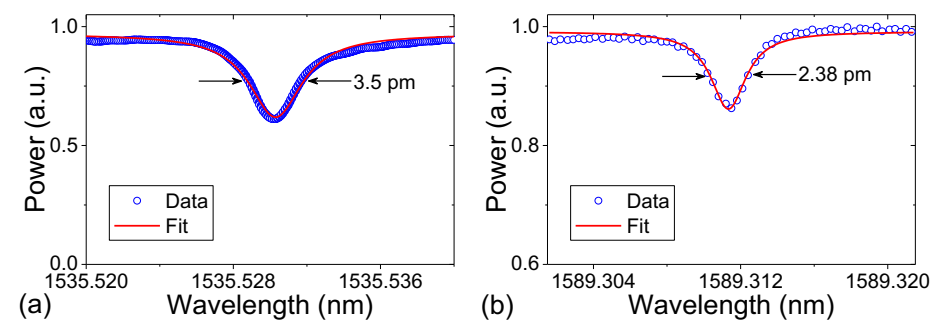

Figure 4 Measured spectrum for fundamental TE mode of 810- $\mu \mathrm{m}$ long AlGaAsOI microresonators with different waveguide cross-section dimensions: (a) $320 \times 630 \mathrm{~nm}^{2}$, (b) $270 \times 900 \mathrm{~nm}^{2}$.

\section{ENGINEERED DISPERSIONS FOR WAVEGUIDES AND MICRORESONATORS}

\subsection{Dispersion in AlGaAsOI waveguides}

The strong light confinement of AlGaAsOI waveguides enables efficient dispersion engineering, which is of critical importance to achieve broadband and efficient nonlinear processes such as FWM, self-phase modulation (SPM), supercontinuum generation and frequency comb generation. Since the waveguide dispersion dominate over the material dispersion, it is seen from Figure 5(a) that anomalous dispersion can be obtained for the fundamental TE mode in spite of a normal dispersion for bulk AlGaAs material. Besides, the dispersion curve can be tailored by changing the waveguide width. Therefore, for a specific pumping wavelength in FWM, one can optimize the phase matching condition to achieve a broadband operation. Figure 5(b) shows the measured FWM conversion efficiency (normalized) as a function of signal wavelengths with pumping at $1567 \mathrm{~nm}$. It is seen that the 660 -nm wide waveguide has the lowest GVD value at the pump wavelength and thus exhibits the widest FWM conversion bandwidth of around $160 \mathrm{~nm}$ covering the whole C- and L-band, which is suitable for ultra-fast optical signal processing such as wavelength conversions [18], [19] and phase-sensitive amplification [20].
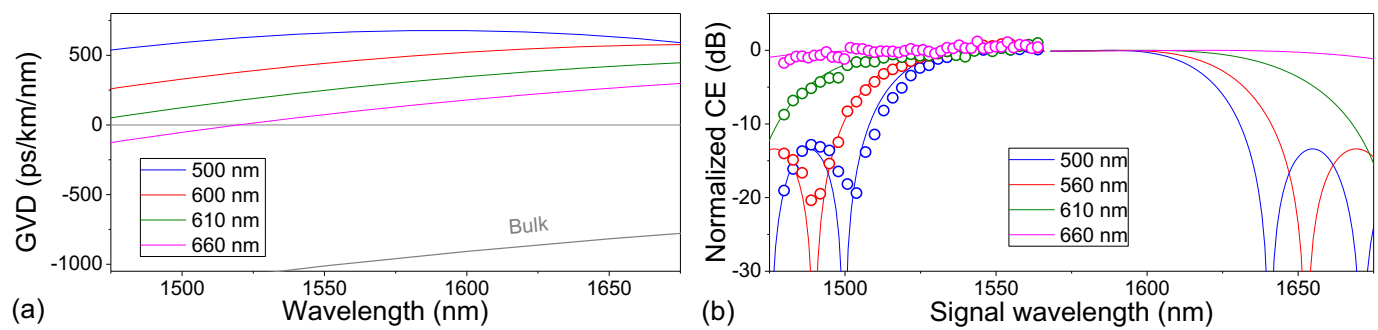

Figure 5 (a) calculated group velocity dispersion (GVD) versus wavelength for 304-nm-thick AlGaAsOI waveguides with different width for the fundamental TE modes. The gray solid curve shows the GVD for the bulk AlGaAs material. (b) Measured FWM conversion efficiency (normalized) for the TE mode by using a continuous wave (CW) pump at $1567 \mathrm{~nm}$. Solid lines are simulated results.

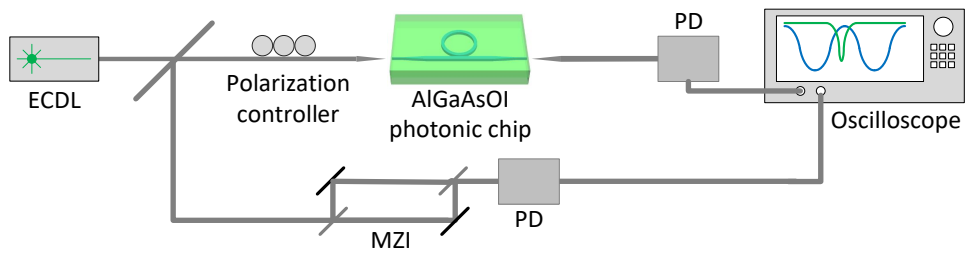

Figure 6 Dispersion measurement setup for AlGaAsOI microresonators. 


\subsection{Dispersion in AlGaAsOI microresonators}

To characterize the dispersion of microresonator devices, the calibrated laser scan setup depicted in Figure 6 is used. An external cavity diode laser (ECDL, Ando AQ4321A) is scanned over $100 \mathrm{~nm}$ range around $1550 \mathrm{~nm}$. The transmitted light through the device is detected by a photodiode (PD) and recorded by an oscilloscope, while part of the ECDL light passes through a free-space Mach-Zehnder Interferometer (MZI) device to calibrate the laser scan rate. The recorded MZI signal is used to construct a calibrated frequency axis that is used to fit the resonances with a theoretical resonator transmission model to calculate the Q factor, and to find the change of the resonator FSR with wavelength to calculate the resonator dispersion.

Microresonator dispersion can be expressed as the deviation of the modes frequencies from an equidistant grid $D_{\text {int }}=\omega_{\mu^{-}}$ $\omega_{0}-\mu D_{l}$, where $\mu$ is the mode number, $\omega_{0}$ is the pumped angular frequency and $D_{l}$ is the free spectral range (FSR) at the pump frequency. As seen in Figure 7, the measured integrated dispersion for $810-\mu \mathrm{m}$ long microresonators with different cross-section dimensions shows anomalous and normal dispersion, respectively.
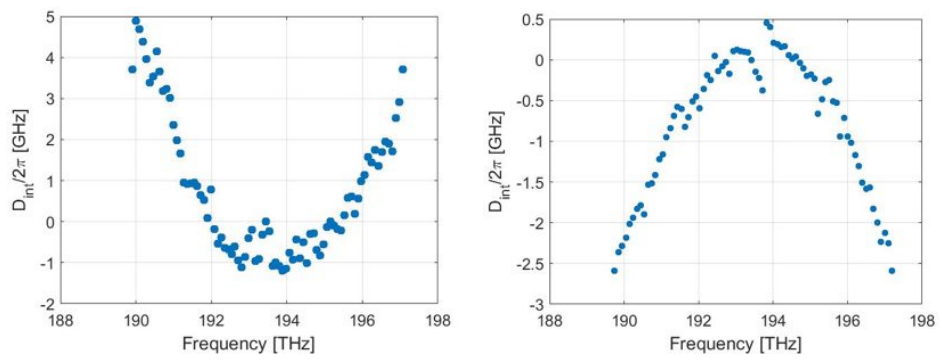

Figure 7 (a) Dispersion of a device with 400nm height and 530nm width. (b) 290nm height and 730nm width.

\section{FREQUENCY COMB GENEARTION}

Frequency combs have broad applications in metrology, spectroscopy, communication, and microwave photonics [21], [22]. Frequency combs can be generated in nonlinear waveguides by spectral broadening mode-locked pulses (or narrow frequency comb) or in microresonators through optical parametric oscillation (OPO) which relies on a combination of parametric amplification and oscillation as a result of the nonlinear FWM processes with presence of a continuous-wave (CW) pumping. In both cases, anomalous dispersion is required for the waveguide.
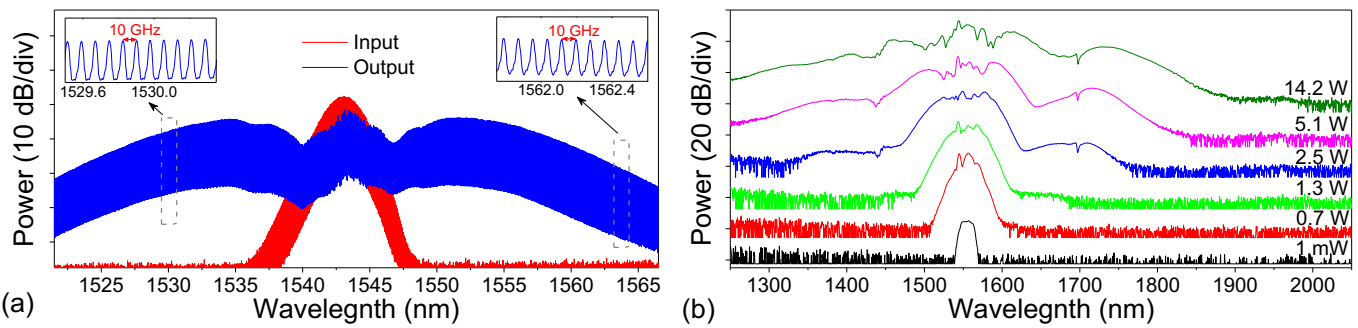

Figure 8 (a) A 10-GHz 1.5-ps pulse generated from a mode-locked laser is launched into the AlGaAsOI nano-waveguide, with an average launched power of only $85 \mathrm{~mW}$. (b) Measured optical spectrum at the output of the AlGaAsOI waveguide with increasing coupled peak pump power. The pulses are with 410 -fs wide and repetition rate is $1 \mathrm{GHz}$.

Figure 8 shows a broadband frequency comb is generated in a nonlinear AlGaAsOI waveguide by SPM-based spectral broadening of $10-\mathrm{GHz}$ picosecond pulses generated from a mode-locked laser. The average launched power is only $85 \mathrm{~mW}$, and the bandwidth of the frequency comb has been increased from about $6 \mathrm{~nm}$ to $44 \mathrm{~nm}$, covering more than the telecom $\mathrm{C}$ band. The insets shows that all comb lines are equidistantly spaced by $10 \mathrm{GHz}$, following the repetition rate of the seed laser and therefore can fit with ITU grid in the telecom communication system allowing for realization a chipbased multi-wavelength light source for WDM transmitters [23]. To further increase the bandwidth of the broadened frequency comb, pulses with low repetition rate can be used and Figure 8 (b) shows the spectrum evolution as the pump power is increased for 1-GHz sub-picosecond pulses [24]. Two modulation instability (MI) sidebands are observed because of the parametric amplification of background noise. In addition, we obtain a continuum spectrum with a $30-\mathrm{dB}$ 
bandwidth of $\sim 500 \mathrm{~nm}$. It is also possible to push the continuum bandwidth to one octave if femtosecond pulses are used and dispersion is further optimized.

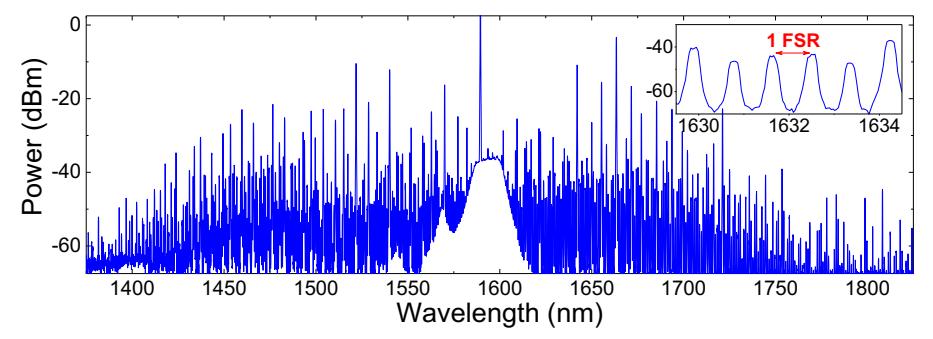

Figure 9 Measured comb spectrum for an 810-um long AlGaAsOI microresonators with anomalous dispersion.

For microcombs, the threshold power for comb generation is inversely proportional to the nonlinear parameter $\gamma$ and the square of the quality factor $(Q)$ of a microresonator. Although significant efforts have been made to improve $Q$ for microresonators in different platforms, the pump powers in most of the demonstrations are at a level beyond the reach of available on-chip laser sources. Thanks to the ultra-high effective nonlinearity of the AlGaAsOI waveguides, we have achieved milli-watt level threshold [13] using a microresonator with a loaded $Q$ of $10^{5}$.

\section{CONCULSION}

We developed AlGaAsOI platforms (AlGaAs-on-silica and AlGaAs-on-sapphire) for nonlinear photonics. Due to the strong light confinement of AlGaAsOI waveguides, an ultra-high nonlinear parameter $\left(660 \mathrm{~W}^{-1} \mathrm{~m}^{-1}\right)$ has been obtained. The high-index contrast layout also makes an efficient dispersion engineering, which is favorable for both $\chi^{(2)}$ and $\chi^{(3)}$ nonlinear processes. The demonstrated results in signal processing and generation show great potential of AlGaAsOI waveguide platforms in various nonlinear applications.

\section{ACKNOWLEDGEMENTS}

This work was supported by the DNRF Research Centre of Excellence, SPOC (DNRF123), and the Villum Foundation's Centre of Excellence, NATEC II

\section{REFERENCES}

[1] G. I. STEGEMAN et al., "AlGaAs BELOW HALF BANDGAP: THE SILICON OF NONLINEAR OPTICAL MATERIALS," J. Nonlinear Opt. Phys. Mater., vol. 3, no. 3, pp. 347-371, Jul. 1994.

[2] J. S. S. Aitchison, D. C. C. Hutchings, J. U. U. Kang, G. I. I. Stegeman, and A. Villeneuve, "The nonlinear optical properties of AlGaAs at the half band gap," IEEE J. Quantum Electron., vol. 33, no. 3, pp. 341-348, Mar. 1997.

[3] K. Dolgaleva, W. C. Ng, L. Qian, and J. S. Aitchison, "Compact highly-nonlinear AlGaAs waveguides for efficient wavelength conversion," Opt. Express, vol. 19, no. 13, pp. 12440-55, Jun. 2011.

[4] C. Lacava, V. Pusino, P. Minzioni, M. Sorel, and I. Cristiani, "Nonlinear properties of AlGaAs waveguides in continuous wave operation regime," Opt. Express, vol. 22, no. 5, p. 5291, Mar. 2014.

[5] J. J. Wathen, P. Apiratikul, C. J. K. Richardson, G. A. Porkolab, G. M. Carter, and T. E. Murphy, "Efficient continuous-wave four-wave mixing in bandgap-engineered AlGaAs waveguides.," Opt. Lett., vol. 39, no. 11, pp. 3161-4, Jun. 2014.

[6] M. Savanier et al., "Large second-harmonic generation at $155 \mu$ min oxidized AlGaAs waveguides," Opt. Lett., vol. 36, no. 15, p. 2955, Aug. 2011.

[7] C. Ozanam et al., "Toward an AlGaAs/AlOx near-infrared integrated optical parametric oscillator," J. Opt. Soc. Am. B, vol. 31, no. 3, p. 542, Mar. 2014.

[8] M. Volatier, D. Duchesne, R. Morandotti, R. Arès, and V. Aimez, "Extremely high aspect ratio GaAs and GaAs/AlGaAs nanowaveguides fabricated using chlorine ICP etching with N2-promoted passivation.," 
Nanotechnology, vol. 21, no. 13, p. 134014, Apr. 2010.

[9] G. A. Porkolab, P. Apiratikul, B. Wang, S. H. Guo, and C. J. K. Richardson, "Low propagation loss AlGaAs waveguides fabricated with plasma-assisted photoresist reflow," Opt. Express, vol. 22, no. 7, p. 7733, 2014.

[10] D. Rafizadeh et al., "Waveguide-coupled AlGaAs/GaAs microcavity ring and disk resonators with high finesse and 216-nm free spectral range," Opt. Lett., vol. 22, no. 16, p. 1244, Aug. 1997.

[11] J. E. Heebner et al., "Enhanced linear and nonlinear optical phase response of AlGaAs microring resonators," Opt. Lett., vol. 29, no. 7, p. 769, 2004.

[12] P. Kultavewuti, V. Pusino, M. Sorel, and J. Stewart Aitchison, "Low-power continuous-wave four-wave mixing wavelength conversion in AlGaAs-nanowaveguide microresonators," Opt. Lett., vol. 40, no. 13, p. 3029, Jul. 2015.

[13] M. Pu, L. Ottaviano, E. Semenova, and K. Yvind, "Efficient frequency comb generation in AlGaAs-oninsulator," Optica, vol. 3, no. 8, p. 823, Aug. 2016.

[14] L. Ottaviano, M. Pu, E. Semenova, and K. Yvind, "Low-loss high-confinement waveguides and microring resonators in AlGaAs-on-insulator," Opt. Lett., vol. 41, no. 17, p. 3996, 2016.

[15] J. Matsuoka, N. Kitamura, S. Fujinaga, T. Kitaoka, and H. Yamashita, "Temperature dependence of refractive index of SiO2 glass," J. Non. Cryst. Solids, vol. 135, no. 1, pp. 86-89, Oct. 1991.

[16] H. K. Sahoo, L. Ottaviano, Y. Zheng, O. Hansen, and K. Yvind, "Low temperature bonding of heterogeneous materials using $\mathrm{Al} 2 \mathrm{O} 3$ as an intermediate layer," J. Vac. Sci. Technol. B, Nanotechnol. Microelectron. Mater. Process. Meas. Phenom., vol. 36, no. 1, p. 11202, Jan. 2018.

[17] J. Leuthold, C. Koos, and W. Freude, "Nonlinear silicon photonics," Nat. Photonics, vol. 4, no. 8, pp. 535-544, Aug. 2010.

[18] M. Pu et al., "AlGaAs-On-Insulator Nanowire with 750 nm FWM Bandwidth, -9 dB CW Conversion Efficiency, and Ultrafast Operation Enabling Record Tbaud Wavelength Conversion," in Optical Fiber Communication Conference (OFC): Postdeadline Paper Digest, 2015, vol. 2015-June, p. Th5A.3.

[19] F. Da Ros et al., "Characterization and Optimization of a High-Efficiency AlGaAs-On-Insulator-Based Wavelength Converter for 64- and 256-QAM Signals,” J. Light. Technol., vol. 35, no. 17, pp. 3750-3757, Sep. 2017.

[20] F. Da Ros et al., "Phase-sensitive four-wave mixing in AlGaAs-on-insulator nano-waveguides," in IEEE Photonics Conference (IPC), 2016, pp. 505-506.

[21] T. J. Kippenberg, R. Holzwarth, and S. A. Diddams, "Microresonator-Based Optical Frequency Combs," Science (80-. )., vol. 332, no. 6029, pp. 555-559, Apr. 2011.

[22] T. Udem, R. Holzwarth, and T. W. Hänsch, "Optical frequency metrology,” Nature, vol. 416, no. 6877, pp. 233237, Mar. 2002.

[23] H. Hu et al., "Single-Source AlGaAs Frequency Comb Transmitter for 661 Tbit/s Data Transmission in a 30core Fiber," in Conference on Lasers and Electro-Optics (CLEO): Postdeadline Paper Digest, 2016, p. JTh4C.1.

[24] M. Pu et al., "Supercontinuum Generation in AlGaAs-On-Insulator Nano-Waveguide at Telecom Wavelengths," in Conference on Lasers and Electro-Optics (CLEO), 2016, p. AM3J.3. 